\title{
Détermination de l'acide butyrique et de l'acide caproïque par une méthode simplifiée
}

\author{
par \\ Khira SPAHIS et Simone KUZDZAL-SAVOIE
}

\section{INTRODUCTION}

La détermination simultanée de l'acide butyrique et de l'acide caproïque présente un intérêt dans divers domaines analytiques : composition de la matière grasse laitière, détermination de la proportion de matière grasse laitière dans un mélange gras, recherche des falsifications du beurre, etc.

En 1968, une méthode a été proposée (Kuzdzal-Savoie et Kuzdzal, Le lait, $\left.\mathrm{n}^{\circ} 475-476,255-260,1968\right)$. Cette méthode repose sur la séparation préliminaire des savons de baryum solubles. Elle s'est révélée utile et a été officialisée en France : arrêté du 19 avril 1973 (Journal Officiel du 5 juin 1973) définissant les " Méthodes officielles d'analyse physique et chimique du beurre ».

Cette méthode présente certains avantages : elle est précise et sûre et, par exemple, s'applique aisément au cas d'étude de mélanges gras ne renfermant qu'une faible proportion de matière grasse laitière. Mais cette méthode présente aussi quelques inconvénients : elle est relativement longue, elle nécessite l'utilisation d'un petit appareillage de verrerie (tube Roos-Kuzdzal) dont la facilité d'emploi n'est pas toujours reconnue ; en outre, un risque de pertes légères existe lors de la dessication des savons si les recommandations concernant les conditions de chauffage ne sont pas respectées.

Dans le présent travail, une méthode simplifiée de détermination de l'acide butyrique et de l'acide caproïque est proposée. Il n'est plus

Laboratoire de Biochimie et Technologie Laitières, INRA-CNRZ - 78350 Jouyen-Josas. 
nécessaire de sécher les savons de baryum. Le tube de Roos-Kuzdzal n'est plus utilisé.

\section{DESCRIPTION DE LA METHODE SIMPLIFIEE DE DETERMINATION DE L'ACIDE BUTYRIQUE ET DE L'ACIDE CAPROIQUE}

\section{Objectif et champ d'application}

La méthode permet de déterminer les quantités respectives d'acide butyrique et d'acide caproïque dans la matière grasse laitière et dans tout mélange de matières grasses contenant de la matière grasse laitière.

\section{Principe}

Saponification de la matière grasse à l'aide d'une solution méthanolique d'hydroxyde de baryum. Récupération des savons d'acides à courte chaîne par filtration. Libération des acides à courte chaîne par l'acide phosphorique. Dosage direct de l'acide butyrique et de l'acide caproïque par chromatographie en phase gazeuse, en présence d'un étalon interne.

\section{Réactifs}

Tous les réactifs doivent être de qualité analytique. L'eau utilisée doit être de l'eau distillée ou une eau de pureté au moins équivalente.

3.1. Acide n-butyrique.

3.2. Acide n-valérique.

3.3. Acide n-caproïque.

3.4. Solution méthanolique d'hydroxyde de baryum environ $0,5 \mathrm{~N}$ : dissoudre par exemple $80 \mathrm{~g}$ d'hydroxyde de baryum $\mathrm{Ba}(\mathrm{OH})_{2}$, $8 \mathrm{H}_{2} \mathrm{O}$ dans $1000 \mathrm{ml}$ de méthanol.

3.5. Acide orthophosphorique : solution aqueuse à $5 \%(\mathrm{v} / \mathrm{v})$.

3.6. Solution étalon : peser à $0,1 \mathrm{mg}$ près $200 \mathrm{mg}$ d'acide $\mathrm{n}$-valérique. Amener à $100 \mathrm{ml}$ avec du méthanol. $1 \mathrm{ml}$ de cette solution correspond à $2 \mathrm{mg}$ d'acide n-valérique.

3.7. Solutions pour la détermination des coefficients de proportionalité :

3.7.1. Préparer une solution aqueuse contenant des quantités définies (égales ou différentes) d'acide n-butyrique et d'acide n-valérique, par exemple $250 \mathrm{mg}$ de chacun des acides dans $100 \mathrm{ml}$ d'eau.

3.7.2. Procéder de la même manière pour préparer la solution d'acide n-caprö̈que et d'acide n-valérique. 
3.8. Solution éthanolique de phénolphtaléine à $1 \%(\mathrm{~m} / \mathrm{v})$ (facultative).

3.9. Produit de remplissage de la colonne : phase stationnaire de type silicone, additionnée de $1 \%$ d'acide phosphorique et imprégnant au taux de $15 \%$ un support de type diatomite lavé à l'acide et de granulométrie 100/120 mesh (voir note 1 ).

\section{Appareillage}

Matériel courant de laboratoire et notamment :

4.1. Tube rodé de $15 \mathrm{~cm}$ de long et d'environ $2 \mathrm{~cm}$ de diamètre (voir note 2).

4.2. Canne de verre, servant de réfrigérant à air.

4.3. Bécher de $25 \mathrm{ml}$.

4.4. Filtre de papier (plissé).

4.5. Agitateur en verre ou spatule.

4.6. Bain d'eau réglé à $75-80^{\circ} \mathrm{C}$.

4.7. Microtubes en plastique de $1,5 \mathrm{ml}$ (voir note 3 ).

4.8. Appareil de chromatographie en phase gazeuse possédant de préférence un détecteur à ionisation de flamme et équipé d'un enregistreur et éventuellement d'un intégrateur.

4.9. Colonne de chromatographie en phase gazeuse en verre ou en acier inoxydable d'environ $2 \mathrm{~m}$ de long et de 1,8 à $2 \mathrm{~mm}$ de diamètre intérieur.

\section{Mode opératoire}

5.1. Prise d'essai.

Peser à $0,1 \mathrm{mg}$ près environ $200 \mathrm{mg}$ de matière grasse dans un tube (4.1.). Ajouter $5 \mathrm{ml}$ de la solution méthanolique d'hydroxyde de baryum (3.4.) et quelques billes de verre. Agiter. Ajouter $2 \mathrm{ml}$ de la solution étalon (3.6.) (dans le cas de la matière grasse laitière) ou moins (dans le cas de mélange de matières grasses). Porter le tube surmonté de la canne de verre (4.2.) dans le bain d'eau (4.6.) et agiter manuellement pendant 2 ou 3 minutes. Maintenir le tube dans le bain d'eau pendant au moins 30 minutes à ébullition douce puis retirer le tube du bain d'eau et refroidir (homogénéiser la suspension à l'aide de l'agitateur en verre [4.5.]). Ajouter 3 à $4 \mathrm{ml}$ d'eau. Homogénéiser à nouveau. Filtrer sur filtre plissé. Recueillir le filtrat dans un bécher de $25 \mathrm{ml}$ (4.3.). Porter celui-ci sur le bain d'eau et l'y maintenir jusqu'à réduction par évaporation à $1 \mathrm{ml}-1,5 \mathrm{ml} \mathrm{du}$ volume du filtrat.

5.2. Libération des acides volatils.

Après avoir soigneusement homogénéisé la solution finale avec une spatule, prélever $0,2 \mathrm{ml}$ de solution dans un microtube (4.7.); ajouter 
$0,15 \mathrm{ml}$ à $0,2 \mathrm{ml}$ de solution d'acide phosphorique (3.5.). Agiter manuellement ou mieux avec un agitateur mécanique (note 4). Laisser reposer 5 à 10 secondes. Prélever à l'aide d'une seringue et à travers le bouchon du microtube 1 à $2 \mu 1$ de la solution aqueuse acide et injecter directement dans l'appareil de chromatographie en phase gazeuse (note 7).

5.3. Analyse des acides gras volatils par chromatographie en phase gazeuse.

L'analyse est effectuée dans des conditions de chromatographie isotherme à une température d'environ $130^{\circ} \mathrm{C}$.

\section{Expression des résultats}

6.1. Détermination des coefficients de proportionnalité de l'acide butyrique et de l'acide caproïque par rapport à l'acide valérique (étalon).

Procéder dans les conditions ci-dessus à l'analyse des solutions aqueuses d'acide butyrique et d'acide valérique d'une part (3.7.1.), et d'acide caproïque et d'acide valérique d'autre part (3.7.2.). Calculer les coefficients de proportionnalité KE4 et KE6 de l'acide butyrique et de l'acide caproïque par rapport à l'étalon (acide valérique) selon les formules suivantes :

$$
\begin{aligned}
\mathrm{KE} 4 & =\frac{\text { aire du pic de l'étalon }}{\text { aire du pic de l'acide butyrique }} \\
\mathrm{KE} 6 & =\frac{\text { aire du pic de l'étalon }}{\text { aire du pic de l'acide caproïque }}
\end{aligned}
$$

si les poids d'acide butyrique, d'acide caproïque et d'acide valérique sont égaux.

Si les poids des acides sont différents utiliser les formules suivantes :

$$
\begin{aligned}
& \mathrm{KE} 4=\frac{\text { poids d'acide butyrique } \times \text { aire du pic de l'étalon }}{\text { poids de l'étalon } \times \text { aire du pic de l'acide butyrique }} \\
& \text { KE6 }=\frac{\text { poids d'acide caproïque } \times \text { aire du pic de l'étalon }}{\text { poids de l'étalon } \times \text { aire du pic de l'acide caproïque }}
\end{aligned}
$$

\subsection{Calcul des résultats.}

Les résultats sont exprimés en grammes d'acide butyrique et d'acide caproïque pour $100 \mathrm{~g}$ de matière grasse.

Les aires des pics sont directement données par l'intégrateur; sinon, les mesurer avec précision. 
Les poids d'acide butyrique et d'acide caproïque sont donnés par les formules suivantes :

$$
\mathrm{X} 4=\frac{100 \times \mathrm{mE} \times \mathrm{A} 4 \times \mathrm{KE} 4}{\mathrm{~m} \times \mathrm{AE}}
$$

et :

dans lesquelles :

$$
\mathrm{X} 6=\frac{100 \times \mathrm{mE} \times \mathrm{A} 6 \times \mathrm{KE} 6}{\mathrm{~m} \times \mathrm{AE}}
$$
tillon ;

$\mathrm{X} 4$ est le poids en grammes d'acide butyrique dans $100 \mathrm{~g}$ d'échantillon ;

X6 est le poids en grammes d'acide caproïque dans 100 g d'échan-

$\mathrm{m}$ est la masse en grammes de l'échantillon;

$\mathrm{mE}$ est la masse en grammes de l'étalon ajouté ;

AE est l'aire intégrée ou mesurée du pic d'acide valérique ;

A4 est l'aire intégrée ou mesurée du pic de l'acide butyrique ;

A6 est l'aire intégrée ou mesurée du pic de l'acide caproïque ;

KE4 est le coefficient de proportionnalité de l'acide butyrique par rapport à l'étalon ;

KE6 est le coefficient de proportionnalité de l'acide caproïque par rapport à l'étalon.

Dans le cas où la présente méthode est utilisée comme complément de la méthode d'analyse des acides gras par chromatographie en phase gazeuse, on considère que l'ensemble des acides à 8 atomes de carbone et plus, représente :

$$
100-(\mathrm{X} 4+\mathrm{X} 6) \text { pour cent des acides totaux }
$$

Dans cette formule :

$\mathrm{X} 4$ représente le poids d'acide butyrique;

X6 représente le poids d'acide caproïque;

$\mathrm{X} 4$ et X6 sont obtenus par la présente méthode.

Notes

Note 1

On trouve dans le commerce des produits de remplissage de colonne prêts à l'emploi, tel que le GP $15 \%$ SP-1220/1 \% H3-PO4 sur chromosorb W AW 100/120 mesh (Supelco Inc.).

Note 2

Les tubes Quickfit MF 24-6 conviennent.

Note 3

Les microtubes en plastique sont jetables et peuvent être trouvés chez Poly-Labo. 


\section{Note 4}

Les agitations et les homogénéisations peuvent être effectuées à l'aide d'un agitateur mécanique, du type Paramix II (Julabo).

\section{Note 5}

Il est nécessaire de rincer soigneusement la seringue avec de l'eau entre deux analyses et, en fin de journée, avec une solution savonneuse diluée pour éviter la corrosion due à l'acide phosphorique.

\section{Note 6}

Il peut être utile de vérifier l'alcalinité de la solution après saponification. Pour cela ajouter 2 ou 3 gouttes de la solution de phénolphtaléine (3.8.), de préférence sur le précipité restant sur le filtre après avoir retiré le bécher contenant le filtrat.

\section{Note 7}

Il est possible de libérer la totalité des acides volatils solubles. Pour cela porter presque à sec la solution de savons de baryum puis ajouter 0,8 à $1 \mathrm{ml}$ de la solution d'acide phosphorique (3.5.). Homogénéiser et injecter 0,5 à $1 \mu \mathrm{l}$ de la solution aqueuse acide directement dans l'appareil de chromatographie en phase gazeuse.

Note 8

Les petites quantités d'acides insolubles présents dans la solution aqueuse acide ne gênent pas le prélèvement pour injection. Les traces d'alcool méthylique ne gênent pas non plus l'analyse. 